\title{
On the central black hole mass in Mkn 501
}

\author{
F. M. Rieger ${ }^{1,2}$ and K. Mannheim ${ }^{1}$ \\ 1 Institut für Theoretische Physik und Astrophysik der Universität Würzburg, Am Hubland, 97074 Würzburg, Germany \\ e-mail: frieger@uni-sw.gwdg.de; mannheim@astro.uni-wuerzburg.de \\ ${ }^{2}$ Universitäts-Sternwarte Göttingen, Geismarlandstr. 11, 37083 Göttingen, Germany
}

Received 17 June 2002 / Accepted 8 October 2002

\begin{abstract}
We analyse the apparent disagreement between the mass estimates of the central black hole(s) in Mkn 501 based on (i) the observations of the host galaxy, (ii) the high energy (HE) emission mechanism, and (iii) the modulation of the beamed radiation by a black hole $(\mathrm{BH})$ binary system. While method (i) seems to imply a central mass $\gtrsim 5 \times 10^{8} M_{\odot}$, method (ii) suggests a BH mass less than $\simeq 6 \times 10^{7} M_{\odot}$. We critically discuss the estimates inferred from (i) showing that current uncertainties may permit a central mass as low as $\simeq(2-3) \times 10^{8} M_{\odot}$. We demonstrate that in this case the estimates (i) and (ii) might be brought into agreement by assuming a binary $\mathrm{BH}$ system where the jet dominating the HE emission originates from the less massive (secondary) BH as suggested by method (iii). On the other hand, if Mkn 501 has in fact a high central BH mass of order $10^{9} M_{\odot}$, a change of fundamental assumptions seems to be required in the context of several HE emission models. We show, that in this case a binary scenario following (iii) may be still possible if the jet which dominates the emission emerges from the more massive (primary) $\mathrm{BH}$ and if the binary evolution passes through phases of super-Eddington accretion and/or decreased conversion efficiency.
\end{abstract}

Key words. BL Lacertae objects: individual: Mkn 501 - galaxies: jets - black hole physics

\section{Introduction}

According to the commonly accepted paradigm, Active Galactic Nuclei (AGN) are thought to harbour supermassive black holes $(\mathrm{BH})$ surrounded by geometrically thin accretion disks, the latter triggering the formation of relativistic jets. Increasing evidence now indicates, however, that this picture probably has to be expanded: first, hierarchical galaxy evolution schemes suggest, that as a result of mergers between galaxies, binary BH systems (BBHSs) should be generally expected in the center of elliptical galaxies, and hence, for example, in the typical hosts of BL Lac objects (cf. Begelman et al. 1980; Kauffmann 1996; Richstone et al. 1998; Milosavljevic \& Merritt 2001; Yu 2002). Secondly, from a phenomenological point of view, a multitude of observational evidence has been plausibly related to the presence of BBHSs in AGN, including the observed misalignment, precession and wiggling of extragalactic jets (e.g. Conway \& Wrobel 1995; Kaastra \& Roos 1992), periodic optical outburst events as observed in the quasar OJ 287 (e.g. Sillanpää et al. 1988; Lehto \& Valtonen 1996; Villata et al. 1998) and the helical motion of knots (e.g. Abraham \& Carrara 1998; Britzen et al. 2001).

One expects that a binary framework for AGN may be particularly relevant for our understanding of BL Lac objects such as Mkn 501 (e.g. De Paolis et al. 2002).
Mkn $501(z=0.034)$ is one of at least four AGN which have been reliably detected at $\mathrm{TeV}$ energies (see Catanese \& Weekes 1999 for a review). From optical observations its host is known to be the elliptical galaxy UGC 10599 (cf. Stickel et al. 1993). As a BL Lac object, Mkn 501 belongs to the blazar class of AGN which are thought to have relativistic jets oriented at a small angle to the line of sight, so that the observed flux is strongly enhanced by relativistic beaming effects. Detailed observations since 1997 have confirmed the picture of powerful activity in Mkn 501 and revealed a wide range of variability patterns during outbursts (e.g. Protheroe et al. 1998) including flaring episodes of several days and rapid variability on subhour timescale (e.g. Sambruna et al. 2000). The evidences for a 23-day periodicity in both the $\mathrm{TeV}$ and the X-ray lightcurves during the 1997 high state (cf. Hayashida et al. 1998; Kranich et al. 1999; Nishikawa et al. 1999) may be counted among the most fascinating features (see Kranich 2001 and Kranich et al. 2001 for a recent assessment of significance) and possibly indicates the presence of a BBHS in Mkn 501 (cf. Rieger \& Mannheim 2000). In this paper we analyse the implications of the apparent disagreement of independent central mass estimates for Mkn 501. Starting with an investigation of the constraints given by host galaxy observations (Sect. 2), we proceed with an analysis of the robustness of the HE emission estimates (Sect. 3) and finally consider the consequences expected from a binary BH modelling (Sect. 4). 


\section{Central mass estimates derived from host galaxy observations}

Recent mass estimates using host galaxy observations mainly rely on the following two statistical correlations:

(1) Dynamical studies of nearby elliptical galaxies have revealed an apparent, almost linear correlation (albeit with significant intrinsic scatter) between the central $\mathrm{BH}$ mass and the $B$-band luminosity of the bulge part of the host galaxy, which is given by $M_{H} \simeq 0.78 \times 10^{8} M_{\odot}\left(L_{B \text {, bulge }} / 10^{10} L_{\odot}\right)^{1.08}$ (Magorrian et al. 1998; Kormendy \& Gebhardt 2001, hereafter KG01).

(2) A much tighter correlation $M_{H} \propto \sigma^{\alpha}$ seems to exist between the BH mass $M_{H}$ in nearby inactive galaxies and the stellar velocity dispersion $\sigma$ of their host bulge (Gebhardt et al. 2000; Ferrarese \& Merritt 2000). However, up to now there is considerable debate over the true slope $\alpha$. Using different samples, Gebhardt et al. (2000) found $\alpha=3.75 \pm 0.3$, while Ferrarese $\&$ Merritt (2000) obtained $\alpha=4.8 \pm 0.54$ and $\alpha=4.72 \pm 0.36$ (Merritt \& Ferrarese 2001a). Currently, further research is required to settle the question whether this difference is mostly caused by lower quality data and a less precise regression algorithm (cf. Merritt \& Ferrarese 2001a) or by systematic differences in the velocity dispersions used by the different groups for the same galaxies (cf. Tremaine et al. 2002).

The results using reverberation mapping (RM) (e.g. Kaspi et al. 2000; Nelson 2000; Wandel 2002) indicate that the $M_{H}-\sigma$ correlation may also hold for nearby AGN. However, a critical test of this conclusion depends on both a secure measure of the $\mathrm{BH}$ mass and an accurate determination of the stellar velocity dispersion. So far, the quality of BH mass estimates from stellar or gas kinematical data (whether ground- or HST-based), which require the $\mathrm{BH}$ sphere of influence $r_{H}=G M_{H} / \sigma^{2}$ to be wellresolved, seems to increase only modestly (Merritt \& Ferrarese 2001a), so that over-estimation may be quite possible. Further progress has been expected using RM methods (Ferrarese et al. 2001). Yet, the accuracy of RM may be strongly affected by systematic errors, e.g. due to uncertainties in the geometry and kinematics of the BLR or due to an unknown angular radiation pattern of the line emission, which may result in a systematic error up to at least a factor of 3 (cf. Krolik 2001). Moreover, only few accurate measurements of $\sigma$ seem to exist for AGN. Ferrarese et al. (2001) have recently analysed six AGN with well-determined RM BH masses by a careful measurement of their velocity dispersions and found a general consistency with the $M_{H}-\sigma$ relation for quiescent galaxies. However, only $\mathrm{BH}$ masses below $\simeq 10^{8} M_{\odot}$ have been included so far, thus leaving out the high mass end of the correlation, and in addition, a large scatter is indicated. Besides providing a promising tool for the determination of $\mathrm{BH}$ masses in $\mathrm{AGN}$, the current uncertainties in the correlations should be considered, if one tries to assess its implication for individual sources such as Mkn 501.

In the case of Mkn 501, Barth et al. (2002a) have recently determined the stellar velocity dispersion from the calcium triplet lines to be $\sigma=(372 \pm 18) \mathrm{km} \mathrm{s}^{-1}$ (cf. also Barth et al. 2002b). Applying the $M_{H}-\sigma$ relations of KG01 and Merritt \& Ferrarese (2001a), they derived a BH mass for Mkn 501 of $M_{H} \simeq(0.9-3.4) \times 10^{9} M_{\odot}$. This mass estimate was supported by the study of $\mathrm{Wu}$ et al. (2002), who estimated the velocity dispersions and $\mathrm{BH}$ masses from the fundamental plane for ellipticals for a large AGN sample including 63 BL Lac objects (but not Mkn 501). They derived BH masses up to $10^{9} M_{\odot}$, but with a potential error up to a factor of two. In particular, inspection of the fit in their Fig. 1 indicates a possible $\mathrm{BH}$ mass for Mkn 501 of $\sim(4-7) \times 10^{8} M_{\odot}$ for $M_{R}$ (host) $=-24.2 \mathrm{mag}$ (Pursimo et al. 2002). The general challenge of determining $\sigma$ accurately may be illustrated in more detail with reference to the recent work by Falomo et al. (2002), who provided a systematical study of the stellar velocity dispersion in seven BL Lacs. Using measurements in two spectral ranges, they found a velocity dispersion of $\sigma=(291 \pm 13) \mathrm{km} \mathrm{s}^{-1}$ for Mkn 501, which is significantly lower than the one derived by Barth et al. (2002a). Hence, if this value is used instead, the BH mass estimated by Barth et al. (2002a) is reduced by up to a factor of three, i.e. one obtains $M_{H} \simeq(3.6-10.7) \times 10^{8} M_{\odot}$. Additional support for such a low $\sigma$-value in Mkn 501 seems to be indicated by the original Faber \& Jackson relation, which yields $\sigma \sim 270 \mathrm{~km} \mathrm{~s}^{-1}$ (see Fig. 2 in Falomo et al. 2002) for $M_{R}$ (host) $=-24.2$ mag. Future research is needed to test whether the discrepancy in $\sigma$ is mainly induced by the difference in the method deriving $\sigma$ (direct fitting versus Fourier quotient routine).

As noted above, an additional mass estimate for Mkn 501 can also be derived from the $M_{H}-L_{\text {bulge }}$ correlation. The reported large uncertainties in this relation have recently been examined by McLure \& Dunlop (2002) using $R$-band luminosities, which are less sensitive to extinction. By analysing the virial $\mathrm{BH}$ masses for a sample of $72 \mathrm{AGN}$, they found the scatter to be quite smaller than previously estimated and stressed its usefulness. For application to Mkn 501, we may exploit the absolute $R$-band luminosity of its host galaxy recently derived by Pursimo et al. (2002) (see also Nilsson et al. 1999). Assuming $H_{0}=50 \mathrm{~km} \mathrm{~s}^{-1} \mathrm{Mpc}^{-1}$, they obtained $M_{R}($ host $)=-24.2 \mathrm{mag}$. If we convert $R$ - to $B$-band luminosity assuming $B-R=1.56$ (e.g. Goudfrooij et al. 1994; Fukugita et al. 1995; Urry et al. 2000), we have $M_{B}$ (host) $=-22.46 \mathrm{mag}$, which results in $L\left(M_{B}(\right.$ host $\left.)\right)=1.406 \times 10^{11} L_{\odot}$. Using the KG01-relation for the $B$-band luminosity, the expected BH mass in Mkn 501 is $M_{H} \simeq 1.3 \times 10^{9} M_{\odot}$, but with a potential error of up to at least a factor of three. Using the more recent McLure \& Dunlop (2002)-relation $\log \left(M_{H} / M_{\odot}\right)=-0.5 M_{R}-2.96( \pm 0.48)$, one finds $M_{H} \simeq(0.46-4.2) \times 10^{9} M_{\odot}$. Uncertainties in the determination of $M_{R}$ may further reduce the expected BH mass. For example, values from the literature presented in Table 4 of Nilsson et al. (1999) indicate that $M_{R}$ might be up to $0.4 \mathrm{mag}$ higher and therefore $M_{H}$ correspondingly smaller. More importantly, if a Hubble constant $H_{0}=70 \mathrm{~km} \mathrm{~s}^{-1} \mathrm{Mpc}^{-1}$ is assumed, one finds $M_{R}=-23.47$, which results in $M_{H} \simeq 7.8 \times 10^{8} M_{\odot}$ (KG01), again with substantial scatter of up to at least a factor of three, or $M_{H} \simeq(2-18) \times 10^{8} M_{\odot}$ (McLure \& Dunlop 2002), thus allowing for a central mass as low as $2 \times 10^{8} M_{\odot}$.

\section{Mass estimates derived in the context of high energy emission models}

Following a quite different approach, the BH mass of Mkn 501 could also be estimated in the framework of high energy 
emission models:

(1) With respect to the high energy emission, Fan et al. (1999) have recently determined the central black hole masses for several $\gamma$-ray loud blazars (including Mkn 501) by assuming that the observed $\gamma$-rays are produced at $\simeq 200 R_{\mathrm{g}}$. Accordingly, the central $\mathrm{BH}$ mass obeys the relation $M_{H} / M_{\odot} \simeq 500 \delta \Delta t$, where $\Delta t$ denotes the doubling timescale in seconds and $\delta$ the Doppler factor. For Mkn 501 they obtained a central BH mass of $M_{H} \simeq 0.9 \times 10^{7} M_{\odot}$, using $\Delta t=6 \mathrm{hrs}$ and $\delta \simeq 0.9$. However, current evidence indicates that the relevant timescale $\Delta t$ might be substantially smaller. Recent observations reveal considerable sub-hour variability (e.g. Ghosh et al. 2000; Catanese \& Sambruna 2000) on a timescale as low as $\Delta t=1200 \mathrm{~s}$ (Sambruna et al. 2000). Such a low value for the observed timescale could possibly be accommodated by assuming a high doppler boosting factor $\delta \simeq 18$. Hence, it appears that the more crucial point in this derivation is the assumption that the $\gamma$-rays dominating the emission are produced at $\sim 200$ Schwarzschild radii. Indeed, at least in the case of the TeV-blazars, the variable, high energy emission is usually regarded as produced by moving knots or shocks in the jet far from the accretion disk (for a review, cf. Mannheim 1997; Aharonian \& Völk 2001 and references therein). While instructive, the derived estimate should thus not be considered as a robust, general limit.

(2) A further mass estimate for Mkn 501 has been derived by DeJager et al. (1999) following an approach developed by Hayashida \& Miyamoto et al. (1998). Assuming the variation in the accretion process to drive the $\mathrm{X}$-ray and $\mathrm{TeV}$ variation in the jet via the dynamo effect, their result yields a central $\mathrm{BH}$ mass of $M_{H}=(1-6) \times 10^{7} M_{\odot}$ for $\delta=(10-15)$. However, due to the absence of a physical basis for the required scaling of the Fourier spectrum and due to the assumption of a linear proportionality between variability timescale and $\mathrm{BH}$ mass (cf. Hayashida et al. 1998), which is probably not valid for the blazar class (Kataoka et al. 2001), this estimate again does not appear to be robust.

(3) The estimates (1) and (2) which suggest a BH mass less than $\sim 6 \times 10^{7} M_{\odot}$, are strongly model-dependent as shown above. We may illustrate this in more detail by comparing them with results derived in the context of another, high energy emission model. Bednarek et al. (1996) for example, have developed a special model for the origin of the high energy particles in TeV blazars like Mkn 421 and Mkn 501, assuming the electrons responsible for the high energy emission to be accelerated rectilinearly in an electric field. In this model, the mass of the central $\mathrm{BH}$ is expected to be limited by $M_{H} \gtrsim 10^{8} M_{\odot}\left(E_{\max } / 34 \mathrm{TeV}\right)^{2.5} / l_{\text {Edd }}^{1 / 2}$, where $E_{\max }$ denotes the maximum photon energy and $l_{\text {Edd }}$ the disk luminosity in units of the Eddington luminosity. There is strong evidence for a sub-Eddington accretion mode in BL Lacs in general (e.g. Cavaliere \& D'Elia 2002) and particularly for the TeV emitting blazars (e.g. Celotti et al. 1998). Thus, using characteristic values, i.e. $l_{\mathrm{Edd}}=(0.01-0.001)$ and $E_{\max }=$ $20 \mathrm{TeV}$ (cf. Samuelson et al. 1998; Konopelko 1999), we arrive at a mass $M_{H} \gtrsim(2.46-14.66) \times 10^{8} M_{\odot}$, which is up to ten times larger than the estimates (1) and (2).

\section{Mass estimates derived in the context of a binary black hole scenario}

In a recent contribution (Rieger \& Mannheim 2000, hereafter RMI; 2001), we have shown that the periodicity of $P_{\mathrm{obs}}=$ 23 days, observed during the 1997 high state of Mkn 501, could be plausibly related to the orbital motion in a BBHS, provided the jet, which dominates the observed emission, emerges from the less massive (secondary) $\mathrm{BH}$. If such an interpretation (henceforth called the standard scenario) is appropriate, we may derive a third estimate for the central mass in Mkn 501. We have demonstrated in RMI that, due to relativistic effects, the observed period appears drastically shortened, so that for the intrinsic Keplerian orbital period one finds $P_{\mathrm{k}}=(6-14) \mathrm{yrs}$. Taking into account that the observed emission is periodically modulated by differential doppler boosting due to the orbital motion, one may derive a simple equation for the required mass dependence in the standard scenario (cf. RMI, Eq. (8)):

$$
\begin{aligned}
\frac{M}{(m+M)^{2 / 3}}= & \frac{P_{\mathrm{obs}}^{1 / 3}}{(2 \pi[1+z] G)^{1 / 3}} \frac{c}{\sin i} \\
& \times \frac{f^{1 /(3+\alpha)}-1}{f^{1 /(3+\alpha)}+1}\left(1-\frac{v_{z}}{c} \cos i\right)^{2 / 3} .
\end{aligned}
$$

Here $\alpha$ denotes the spectral flux index, $z$ the redshift, $v_{z}$ the outflow velocity, $i \simeq 1 / \Gamma_{\mathrm{b}}$ the angle of the jet axis to the line of sight, $\Gamma_{b} \simeq 10-15$ (e.g. Mannheim et al. 1996; Spada et al. 1999) the bulk Lorentz factor and $f$ the observed flux ratio between maximum and minimum (for the TeV range $f \sim 8$ ), while $m$ and $M$ denote the masses of the smaller and larger $\mathrm{BH}$, respectively.

In order to break the degeneracy in this mass ratio, we may utilize an additional constraint by assuming that the current binary separation $d$ corresponds to the separation at which gravitational radiation becomes dominant (cf. RMI). Such a constraint yields an upper limit for the allowed binary masses and might be associated with the key aspect that BL Lac objects are old, more evolved and underluminous sources, i.e. they might be close binaries, probably settled above or near the critical gravitational separation, because the possibility of removing further angular momentum has been almost terminated as a result of declining gas accretion rates. We can specify the corresponding gravitational separation $d_{\mathrm{g}}$ by equating the timescale $\tau_{\text {grav }}=|d / \dot{d}|=\left(5 c^{5} / 64 G^{3}\right) d^{4} /(M m[m+M])$ on which gravitational radiation shrinks the binary orbit, with the dynamical timescale $\tau_{\text {gas }}$ for gas accretion (cf. Begelman et al. 1980; note that compared with RMI, this estimate for $\tau_{\text {grav }}$ is a factor 2.5 more precise, cf. Rieger \& Mannheim 2001). A characteristic measure for $\tau_{\text {gas }}$ is given by the Eddington limit $\tau_{\text {gas }} \simeq 3.77 \times 10^{7}(\eta / 0.1)$ yrs, assuming a canonical $10 \%$ conversion efficiency (cf. Krolik 1999). Using $\tau_{\text {grav }}$ and $\tau_{\text {gas }}$, one finally arrives at

$d_{\mathrm{g}} \simeq 3.50 \times 10^{16} M_{8}^{1 / 4} m_{8}^{1 / 4}\left(m_{8}+M_{8}\right)^{1 / 4} \mathrm{~cm}$.

If we combine this expression with the relevant expression for the binary separation (i.e. Eq. (7) of RMI), we obtain a second constraint on the allowed mass ratio given by

$\frac{\left(m_{8}+M_{8}\right)^{3 / 4}}{M_{8}^{5 / 4} m_{8}^{1 / 4}}=7.3 \times 10^{6} \frac{f^{1 /(3+\alpha)}+1}{f^{1 /(3+\alpha)}-1} \frac{(1+z)}{P_{\mathrm{obs}}} \sin i$, 


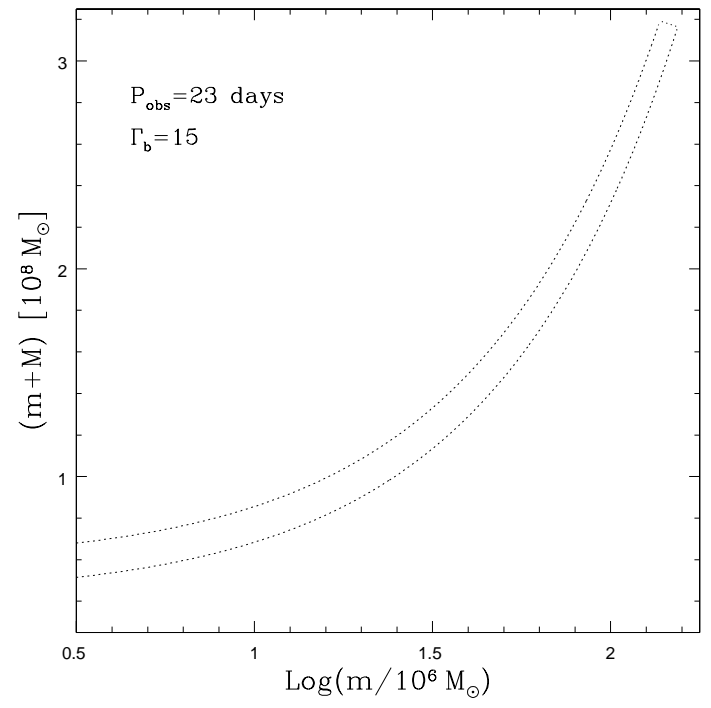

Fig. 1. Allowed central mass dependence as a function of the secondary mass assuming a BBHS, where the observed periodicity of 23 days is related to the orbital motion of a jet, which emerges from the less massive $\mathrm{BH}$. The allowed mass range lies inside the curves and is calculated using $\Gamma_{\mathrm{b}}=15$.

assuming the jet to arise from the less massive $\mathrm{BH}$ (for the reverse case the BH masses should be interchanged in Eqs. (1) and (3)). By using Eqs. (1) and (3), we may determine the binary masses permitted for a BBHS with separation near that for which gravitational radiation becomes dominant. In Fig. 1 we have plotted the allowed range for the case of $\Gamma_{\mathrm{b}}=15$ and $\alpha \simeq$ (1.2-1.7) (e.g. Aharonian et al. 1999). The run of the curves is determined by Eq. (1) with the upper bounds given by Eq. (3). We note that the upper bounds depend on the presumed timescale for the binary evolution, i.e. on the estimate of the gravitational separation. In particular, the derived upper bounds may be larger, if, for example, disk-driven migration (e.g. Armitage \& Natarajan 2002) might be relevant. Figure 1 reveals that (a) the typical combined mass for a BBHS is expected to be less than $\sim 3 \times 10^{8} M_{\odot}$, and that (b) a BBHS with, e.g. $m \sim 6 \times 10^{7} M_{\odot}$ and combined central mass $(M+m) \simeq(1.5-2) \times 10^{8} M_{\odot}$ appears well-conceivable given the current limits.

On the other hand, if a high central mass of $\sim 10^{9} M_{\odot}$ will be established by further research, the proposed binary scenario appears to be ruled out. We note, however, that even in this case a binary scenario may be still possible provided that the jet, which dominates the emission, is produced by the primary $\mathrm{BH}$. To illustrate the implications in this case, let us consider a (combined) central mass of $\simeq 0.9 \times 10^{9} M_{\odot}$ by demanding the primary to be in the range $(5-6) \times 10^{8} M_{\odot}$ (see Table 1). The mass of the secondary BH then is determined by Eq. (1) with the masses interchanged, the current separation $d$ by Eq. (3) of RMI (again with the masses interchanged) and the gravitational separation $d_{\mathrm{g}}$ by Eq. (2). The results as shown in Table 1 imply a close binary system with $d \lesssim d_{\mathrm{g}}$. If the optically bright QSO stage thus occurs during the binary evolution and the applied doppler factors are considered as typical, phases of super-Eddington accretion and/or with decreased
Table 1. Binary masses for a central core mass of $\simeq 0.9 \times 10^{9} M_{\odot}$, currently expected separation $d$ and corresponding gravitational separation $d_{\mathrm{g}}$. The calculations have been done using $\alpha=1.2$ (in brackets: $\alpha=1.7)$. The jet is assumed to be produced by the more massive $\mathrm{BH}$.

\begin{tabular}{lllll}
\hline \hline$i=1 / \Gamma_{\mathrm{b}}$ & $1 / 10$ & & $1 / 15$ & \\
\hline$m\left[10^{8} M_{\odot}\right]$ & 4.22 & $(3.62)$ & 3.85 & $(3.34)$ \\
$M\left[10^{8} M_{\odot}\right]$ & 5.00 & $(5.00)$ & 6.00 & $(6.00)$ \\
$d\left[10^{16} \mathrm{~cm}\right]$ & 4.87 & $(4.76)$ & 8.54 & $(8.39)$ \\
$d_{\mathrm{g}}\left[10^{16} \mathrm{~cm}\right]$ & 13.1 & $(12.4)$ & 13.6 & $(12.9)$ \\
\hline
\end{tabular}

conversion efficiency seem to be necessary for the binary to be above its gravitational separation.

\section{Discussion and conclusions}

In this paper, we have considered the apparent discrepancies between independent central mass estimates for the paradigmatic object Mkn 501. Our main conclusions can be summarized as follows:

(1) Estimates inferred from host galaxy observations using the reported, statistical correlations for the central velocity dispersion and the bulge luminosity reveal a tendency for the central $\mathrm{BH}$ mass in Mkn 501 to be larger than $\sim 5 \times 10^{8} M_{\odot}$. If verified by further investigations, this would exclude both the standard binary scenario, where the jet is emitted from the less massive $\mathrm{BH}$, and several high energy emission models proposed so far. However, as the correlations still show substantial intrinsic scatter and large uncertainties due to the use of limited samples - especially with respect to the high mass end - the accuracy of this estimate should be treated with caution. In particular, we have shown that the central mass limit could be easily up to a factor of (2-3) smaller, thus allowing for the standard binary scenario, in which case a simple explanation for the divergent mass estimates from emission models and host observations appears possible.

(2) Independent mass estimates from high energy emission models suggest a mass for the jet emitting BH in Mkn 501 which is smaller than $\simeq 6 \times 10^{7} M_{\odot}$. However, this estimate is quite model-dependent and so cannot be used as a universally valid constraint. In particular, emission models have been developed for Mkn 501 where a central BH mass up to ten times larger appears quite possible.

(3) If a binary $\mathrm{BH}$ interpretation for the periodical variability (cf. RMI) is appropriate in the case of Mkn 501, the jet dominating the emission has to be produced by the less massive (secondary) BH. Using characteristic jet parameters and assuming both Eddington-limited accretion and a binary separation comparable to the gravitational one, we have shown that the combined central (primary + secondary $\mathrm{BH}$ ) mass should be smaller than $\simeq 3 \times 10^{8} M_{\odot}$. The binary model particularly permits a system with $m=6 \times 10^{7} M_{\odot}$ and $(m+M) \simeq$ $(1.5-2) \times 10^{8} M_{\odot}$, possibly allowing for a convergence of constraints from emission models and host observations.

(4) If further research clearly establishes a high central $\mathrm{BH}$ mass $\sim 10^{9} M_{\odot}$ in Mkn 501, this would call for a change of fundamental assumptions and/or a modification of the 
parameter space regarded to be typical in the context of several high energy emission models. The only way to avoid this conclusion seems to be the assumption of a highly unequal BBHS with the jet dominating the emission produced by the less massive and the central mass dominated by the primary $\mathrm{BH}$. However, it then appears no longer be possible to explain the observed periodicity via the orbital motion. On the other hand, even in the case of a high central mass, a binary model for the observed periodicity may be still possible provided the jet is produced by the primary BH. Yet, for the binary to be near or above the separation at which gravitational radiation becomes dominant, one then requires the earlier binary evolution to pass through phases of super-Eddington accretion and/or decreased conversion efficiency $\eta<0.1$. Such conditions need not necessarily be ad hoc but have already been considered in the context of galaxy evolution and the growth of massive $\mathrm{BH}$ (e.g. Collin et al. 2002; Yu \& Tremaine 2002).

In view of Mkn 501 as a paradigmatic object, bringing its mass estimates to convergence continues to be an important task. Further research on the parameter space of emission models and the careful observations of nearby galaxies will be particularly valuable in order to minimise the uncertainties in the statistical correlations, to evaluate the impact of host observations on high energy emission models, and to assess the plausibility of a BBH model.

Acknowledgements. We are grateful to J. Heidt for fruitful discussions about the host of Mkn 501, and C. Hettlage and R. Hessman for a helpful reading of the manuscript. F.M.R. gratefully acknowledges support under DFG MA 1545/8-1.

\section{References}

Abraham, Z., \& Carrara, E. A. 1998, ApJ, 496, 172

Aharonian, F. A., Akhperjanian, A. G., Barrio, J. A., et al. 1999, A\&A, 349,11

Aharonian, F. A., Völk, H. J. (eds.) 2001, High Energy Gamma-Ray Astronomy, AIP Conf. Proc. 558

Armitage, P. J., \& Natarajan, P. 2002, ApJ, 567, L9

Barth, A. J., Ho, L. C., \& Sargent, W. L. W. 2002a, ApJ, 566, L13

Barth, A. J., Ho, L. C., \& Sargent, W. L. W. 2002b, ApJ, to appear [astro-ph/0209562]

Bednarek, W., Kirk, J. G., \& Mastichiadis, A. 1996, A\&A, 307, L17

Begelman, M. C., Blandford, R. D., \& Rees, M. J. 1980, Nature, 287, 307

Britzen, S., Roland, J., Laskar, J., et al. 2001, A\&A, 374, 784

Catanese, M., \& Weekes, T. C. 1999, PASP, 111, 1193

Catanese, M., \& Sambruna, R. M. 2000, ApJ, 534, L39

Cavaliere, A., \& D'Elia, V. 2002, ApJ, 571, 226

Celotti, A., Fabian, A. C., \& Rees, M. J. 1998, MNRAS, 293, 239

Collin, S., Boisson, C., Mouchet, M., et al. 2002, A\&A, 388, 771

Conway, J. E., \& Wrobel, J. M. 1995, ApJ, 439, 98

De Jager, O. C., Kranich, D., Lorenz, E., \& Kestel, M. 1999, Proc. of 26th ICRC (Salt Lake City), vol. 3, p. 346

De Paolis, F., Ingrosso, G., \& Nucita, A. A. 2002, A\&A, 388, 470

Falomo, R., Kotilainen, J. K., \& Treves, A. 2002, ApJ, 569, L35

Fan, J. H., Xie, G. Z., \& Bacon R. 1999, A\&AS, 136, 13

Ferrarese, L., \& Merritt, D. 2000, ApJ, 539, L9

Ferrarese, L., \& Merritt, D. 2001, ApJ, 555, L79

Ferrarese, L., Pogge, R. W., Peterson, B. M., et al. 2001, ApJ, 555, L79
Fukugita, M., Shimasaku, K., \& Ichikawa, T. 1995, PASP, 107, 945 Gebhardt, K., Bender, R., Bower, G., et al. 2000, ApJ, 539, L13

Ghosh, K. K., Ramsey, B. D., Sadun, A. C., \& Soundararajaperumal, S. 2000, ApJS, 127, 11

Goudfrooij, P., Hansen, L., Jorgensen, H. E., et al. 1994, A\&A, 104, 179

Hayashida, K., Miyamoto, S., Kitamoto, S., et al. 1998, ApJ, 500, 642

Hayashida, N., Hirasawa, H., Ishikawa, F., et al. 1998, ApJ, 504, L71

Kaastra, J. S., \& Roos, N. 1992, A\&A, 254, 96

Kaspi, S., Smith, P. S., Netzer, H., et al. 2000, ApJ, 533, 631

Kataoka, J., Takahashi, T., Wagner, S. J., et al. 2001, ApJ, 560, 659

Kauffmann, G. 1996, MNRAS, 281, 487

Konopelko, A. 1999, Astropart. Phys. 11, 135

Kormendy, J., \& Gebhardt, K. 2001, in 20th Texas Symp. on Relativistic Astrophysics, ed. J. C. Wheeler, \& H. Martel, AIP Conf. Proc., 586, 363 (KG01)

Kranich, D., deJager, O. C., Kestel, M., et al. 1999, Proc. of 26th ICRC (Salt Lake City), vol. 3, p. 358

Kranich, D. 2001, Ph.D. Thesis, Technische Universität München

Kranich, D., deJager, O., Kestel, M., et al. 2001, Proc. of 27th ICRC (Hamburg), vol. 7, p. 2630

Krolik, J. H. 1999, Active Galactic Nuclei (Princeton Univ. Press, Princeton)

Krolik, J. H. 2001, ApJ, 551, 72

Lehto, H. J., \& Valtonen, M. J. 1996, ApJ, 460, 207

Magorrian, J., Tremaine, S., Richstone, D., et al. 1998, AJ, 115, 2285

Mannheim, K., Westerhoff, S., Meyer, H., \& Fink, H.-H. 1996, A\&A, 315,77

Mannheim, K. 1997, in Very High Energy Phenomena in the Universe, ed. Y. Girand-Héraud, \& J. T. Thanh Van, p. 17

McLure, R. J., \& Dunlop, J. S. 2002, MNRAS, 331, 795

Merritt, D., \& Ferrarese, L. 2001a, in The Central Kiloparsec of Starbursts and AGN, ed. J. H. Knapen, J. E. Beckman et al., ASP Conf. Proc., 249, 335

Merritt, D., \& Ferrarese, L. 2001b, ApJ, 547, 140

Milosavljevic, M., \& Merritt, D. 2001, ApJ, 563, 34

Nelson, C. H. 2000, ApJ, 544, L91

Nilsson, K., Pursimo, T., Takalo, L. O., et al. 1999, PASP, 111, 1223

Nishikawa, D., Hayashi, S., Chamoto, N., et al. 1999, Proc. of 26th ICRC (Salt Lake City), vol. 3, p. 354

Pursimo, T., Nilsson, K., Takalo, L. O., et al. 2002, A\&A, 381, 810

Protheroe, J., Bhat, C. L., Fleury, P., et al. 1998, Proc. of 25th ICRC (Durban), vol. 8, p. 317

Richstone, D., Ajhar, E. A., Bender, R., et al. 1998, Nature, 395A, 14

Rieger, F. M., \& Mannheim, K. 2000, A\&A, 359, 948 (RMI)

Rieger, F. M., \& Mannheim, K. 2001, in High Energy Gamma-Ray Astronomy, ed. F. A. Aharonian, \& H. Völk, AIP Conf. Proc., 558,716

Roos, N. 1988, ApJ, 334, 95

Sambruna, R. M., Aharonian, F. A., Krawczynski, H., et al. 2000, ApJ, 538,127

Samuelson, F. W., Biller, S. D., Bond, I. H., et al. 1998, ApJ, 501, L17 Sillanpää, A., Haarala, S., Valtonen, M. J., et al. 1988, ApJ, 325, 628

Spada, M., Salvati, M., \& Pacini, F. 1999, ApJ, 511, 136

Stickel, M. R., Fried, J. W., \& Kühr, H. 1993, A\&AS, 98, 393

Tremaine, S., Gebhardt, K., Bender, R., et al. 2002, ApJ, 574, 740

Urry, C. M., Scarpa, R., O’Dowd, M., et al. 2000, ApJ, 532, 816

Villata, M., Raiteri, C. M., Sillanpää, A., \& Takalo, L. O. 1998, MNRAS, 293, L13

Wandel, A. 2002, ApJ, 565, 762

Wu, X.-B., Liu, F. K., \& Zhang, T. Z. 2002, A\&A, 389, 742

Yu, Q. 2002, MNRAS, 331, 935

Yu, Q., \& Tremaine, S. 2002, MNRAS, 335, 965 